\title{
PRINCIPLES AND MECHANISMS OF BRAIN FUNCTIONING
}

\author{
Vladimir Shumilov * \\ National Research Tomsk State University, 634050, Tomsk, Russia
}

\begin{abstract}
Principles of brain functioning and a mechanism for their realization are formulated in the paper. An absolutely local mechanism provides independent of any external control formation of traces of events in the form of connections between neurons (accumulation of experience), storage of these traces (fading memory), interpretation of traces (response to the impact of the environment in accordance with accumulated experience). Traces of events influence formation of the pathways of signals from the receptors to the effectors and, consequently, the organism's reaction. Retraining is provided by formation of new and therefore stronger links.
\end{abstract}

\section{Introduction}

For over 60 years, researchers have been working on the task of creating artificial intelligence (AI), but this task is still far from the acceptable solution. The information capacity of the brain, where the human intellect is located, is so large (at least 300 terabytes), that hundreds of thousands and millions of years of labor of highly skilled specialists are needed in order to fill in such capacity by information [1]. This circumstance makes creation of AI by today's methods economically inexpedient, although, there are no principal barriers to creation of AI that is not inferior to human intelligence. Required computer power will be achieved in the near future.

However, creation of AI will contribute almost nothing to understanding functioning of the brain of humans and animals. In addition, for the constant development of the created intelligence, it is necessary to ensure its openness, the ability to perceive the flow of information with no less speed than a person does.

\section{The main properties of the natural carrier of intelligence as an information processing system}

The nervous system (NS) and the brain are a natural self-organizing information processing system, which is the system that converts signals about dangerous (harmful) environmental influences on the body generated in receptors into signals that control the mechanisms of evading these impacts on the organism.

*Corresponding author: vnshumilov@rambler.ru 
Historically, the nervous system of biological organisms with receptors, a signal processing system (actually the brain) and effectors (manipulators) has emerged as a means of organisms' survival by means of avoiding dangerous effects. This ensured the survival and expansion of organisms with the NS.

The paths of the signals along the NS include a multitude of neurons and the connections between them in the retransmission mode without generating new signals independently. Neurons provide undamped progress of signals through inter-neural connections. This advance is ensured by excitation of the neuron when a total input signal arriving at its input exceeds a certain excitation threshold. The total input signal represents the common impact on the neuron of previous neurons (or receptors, which are special input neurons) delivering signals to the neuron's input through all of its multiple input inter-neural connections. The neuron is excited by a relatively small input signal. Upon excitation, the neuron generates a powerful signal at the output that participates in the excitation of subsequent neurons through their input connections. This ensures undamped progress of signals through the brain structures (from receptors to effectors).

The energy of a powerful signal is extracted by a neuron from food products delivered to it through the blood. In addition, the functioning of the neuron provides many other functions.

\section{The pace of information processing by the human brain}

It has been proved, that the experience of biological beings is accumulated in the connections between neurons in the corresponding zones of the brain [2]. This occurs with an average rate of forming of connections between neurons in the human brain of about 30,000 bonds per second during a lifetime (the number of possible connections / lifetime) [1]. It is clear, that no centralized mechanism can provide such speed of forming connections in absolutely certain places. Where could it come from? The impossibility of existence of a centralized mechanism to control formation of inter-neural connections in the brain becomes evident if we recall that the development of the organism begins with a single mother cell, which has no place for additional information. This cell may contain only information about the self-duplication of this cell, about possible variations in the generation of new copies of cells depending on the presence of various components (building materials) in the neighborhood of the cell for new cells and the mechanism of cell functioning. On the other hand, even if the central mechanism existed, how would it manage to get a huge amount of information about the place, in which the next inter-neural connection should be established?

\section{A local mechanism that ensures functioning of the brain}

The problem of forming a connection between neurons at the required speed and in the right place is easily solved by the local mechanism proposed by us. We note that the connections between neurons are paired, and they are formed only between two contiguous neurons that are excited at the same time. If this were not true, all possible connections would quickly be formed even between unexcited neurons. Moreover, the connections formed would not correspond to anything. In fact, observations show that the connections between brain's neurons of animals are intensively formed precisely in those areas of the brain that are responsible for the type of activity that the animal is being taught [2].

What mechanism could provide the formation between the simultaneously excited neighboring neurons? Such a mechanism is provided by a neuron, a model of which we propose. In contrast to the classical McCulloch-Pitts perceptron model [3], with which we can illustrate only some aspects of the functioning of artificial neural systems, we propose a 
neuron model, which explains in fact all the basic processes, occurring in the brain. Figure 1 represents a scheme of this model.

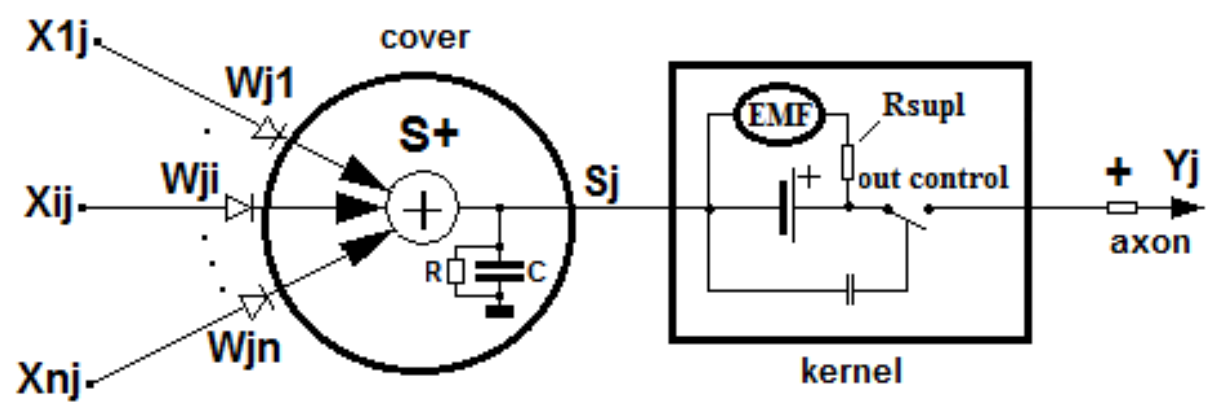

Fig. 1. Model of the neuron, which ensures functioning of the brain.

The key at the neuron output (at the beginning of the axon) can be manipulated in different ways. It can be a thyristor or a field effect transistor, or even just a mechanical shutdown of the ion channel. The answer to this question is not so fundamental and can be obtained later.

The model proposed differs significantly from the classical model because it clearly indicates the source of energy for the formation of a pulse. This impulse is transmitted further by the neuron when it is excited and when the level of the total input signal exceeds the excitation threshold.

The analysis of the above circuit gives an idea of its response to the total input signal. This response is shown in Figure $2 a, 2 b$ with the response of the $\mathbf{H}(\mathbf{S j})$ McCulloch-Pitts perceptron.

Let us explain the Figure. The perceptron forms the response potential as a Heaviside function as soon as the total input signal $\mathbf{S j}$ exceeds a certain threshold $\mathbf{U t}$. The output potential in this model is zero when the input signal drops below the Ut level.

a)

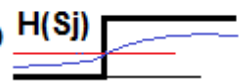

c)



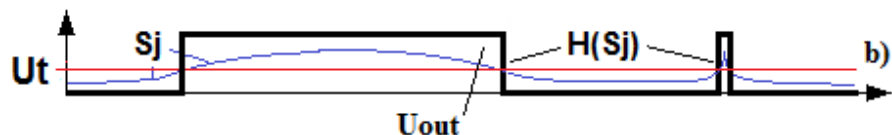

Uout

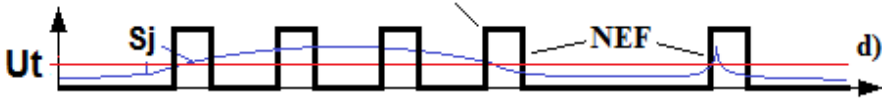

Fig. 2. Excitation function of the McCulloch-Pitts perceptron and the proposed model of the neuron.

The response of our model is significantly different. It is presented in Figure $2 d$. Here, on the vertical axis, the potential difference between the point of the total input signal $\mathbf{S j}$ and the axon of the neuron is reflected.

We call the function depicted in Figure $2 c$ as a Neuron Excitation Function (NEF). Let us consider it. The characteristics of the function are determined by the parameters of the neuron and its current state. The properties of this function are the folowing:

1) For a prolonged $\mathbf{S} \mathbf{j}<\mathbf{U t} \mathbf{N e F}=\mathbf{0}$ (to point 1 on the left). That is, the neuron is not excited (state of rest). 
2) When the conditions $(\mathbf{S} \mathbf{j} \geq \mathbf{U t}) \boldsymbol{\&}\left({ }^{\wedge} \mathbf{R T}\right)$ are satisfied, the neuron is excited (points 1 and 2 in Figure 2c.) A positive pulse with a duration of PD (Pulse Duration) begins, which continues to point 3 .

3) At point 3, the impulse ends because of expending of accumulated energy.

4) At point 3, restoration of the neuron's operability begins, excitation of which has just ended. Recovery lasts for a "dead" time RT (Recovery Time). RT ends at point 4, at the moment of restoring the neuron's ability to get excited. The excitation cycle is complete on this. The neuron is ready for the next excitation. If the value of the input signal $\mathbf{S j}>\mathbf{U t}$ is longer than the duration of one cycle (for example, for 4 cycles), at the neuron output, we will observe not one long pulse, as for the standard Heaviside function, but a series of 4-cycle pulses generated very simple because of presence of "dead" time. The pulse duration PD and the "dead" time RT are determined by the parameters of the neuron (the capacity of the energy store and the time of its charging).

Given that the neuron is electrically isolated rather well but there are leakage currents, we come to a conclusion that the potentials of the inputs, the total input and output of the unexcited neuron are close to 0 ("ground"). In addition, when the neuron is excited, because of the closure of the key, the total input potential $\mathbf{S j}$ switches in the potential difference U2 between the total input and the axon. In electrical terms, a neuron is "suspended" on its inputs and outputs. Therefore, as a result of the appearance of a potential difference between the total input and the axon, the axon potential becomes positive when the neuron is excited, and the potential of the total input becomes negative.

\section{A mechanism of formation of connections between neurons}

From the most general point of view, all natural processes produce some changes. The propagation of signals in the brain from the receptors to the effectors also produces some changes. In the energy sense, the process of signal propagation produces the greatest change in the effector, which is a place of transformation of weak signals into mechanical or chemical transformations. In addition, the propagation of signals through the brain leaves traces of events in the form of connections between neurons formed during the propagation of signals.

After considering the appearance of potentials at the output and input of an excited neuron, it becomes clear how the bonds are formed, and only between excited neurons. Connections between neurons can be formed only when the potential difference between neighboring points of two excited neurons is higher than some bond potential (BP) for formation of a bond. We see that the proposed scheme easily ensures fulfillment of this requirement. Formation of connections between neurons is explained in Figure $3 a$.


b)

Fig. 3. Formation of a new connection made by the potential difference, which is greater than the bond formation potential, and compensation of the potentials of excited neurons.

The potentials $\mathrm{U}+$ and $\mathrm{U}$ - at the output (axon) and at the total input of the excited neuron are individually smaller in modulus than $\mathrm{BP}$, and $\mid \mathrm{U}+$ and $\mathrm{U}-\mid$ are larger than $\mathrm{BP}$. Therefore, a galvanic bond can be formed between adjacent parts of two excited neurons, and communication can be formed only between excited neurons. For example, as a result of 
electrical breakdown (or other irreversible phenomenon), a long-term conducting channel can be formed, which is an inter-neural connection with some initial conductivity (logical weight). Moreover, the conductivity of this channel (logical connection weight) may decrease with time. For example, it happens due to diffusion. This reduction in the weight of links represents a process of forgetting over time.

That is, the neuron model proposed easily provides formation of traces of events affecting the organism that is associative connections between excited neurons, which are a reflection of the organism's experience. Subsequently, formed traces of events or inter-neuronal connections (experience) are involved in formation of the reaction, that is, in formation of the paths of signals propagating from receptors to manipulators. Namely, because of the presence of a connection between neurons excited approximately at the same time with the appearance of one stimulus, a chain of neurons associated with another stimulus will immediately be excited through the previously formed bond. As a result of this induced excitation through the previously formed bond, the organism will react to the second, not yet come, stimulus, as if foreseeing its appearance. Since in nature not random events, but the chains of causally related events occur, thanks to this one-part forecast an organism managed even by only two or three neurons can survive with greater probability than an organism that does not have the ability to fix traces of events that have affected organism. Thus, because of formation of traces of past events in the brain, the past experience influences the body's response to the current impact of the environment on the body. That is the key useful feature of the brain necessary for the survival of the organism and expansion of its species.

Thus, by changing the structure of the brain because of formation of traces of events (new pathways of signals following) the model provides training (fixation of information, storing and interpreting it). Interpretation of the fixed information at the local level looks like signal passing through different paths (the inter-neural connections). From the point of view of technical implementation, this ensures the full functioning of the information processing system. That is, our model ensures fixation, storage and interpretation of the fixed information.

\section{Analysis of the mechanism of neuron interaction}

The input and output circuits of neurons have a finite load capacity. This leads to the fact that the potentials of the inputs and outputs of the excited neurons are mutually compensated, as shown in Figure $3 b$. As a result of compensation of potentials, the first excited neuron prevents the excitation of other neurons because of the reduction (compensation) of the potentials that had caused its excitation. Because of the compensation of potentials, a situation occurs, which in the theory of neural networks is viewed as a requirement and as a principle "The first receives everything". This requirement in our model is a consequence and prevents the self-excitation of the processing system. Self-excitation will occur if the average multiplication factor of signals (K-factor) is greater than 1 . In this case, after each next step in the propagation of signals, their number will grow as $\mathrm{K}$-factor ${ }^{\mathrm{N}}$. K-factor is also regulated within the species evolutionarily, from generation to generation because of the more likely survival of organisms with a suitable neuron parameter set.

Figure $4 a$ shows a scheme for determining the point, at which a connection is formed between the output of an excited neuron (at the top of the Figure) and the input of another excited neuron (in the right part of Figure $4 a$ ). However, in nature there are no regular flat rectangular intersections. The outputs and inputs of neurons are located in a 3-dimensional volume, which provides even more opportunities for formation of connections (Figure $4 b$ ). 

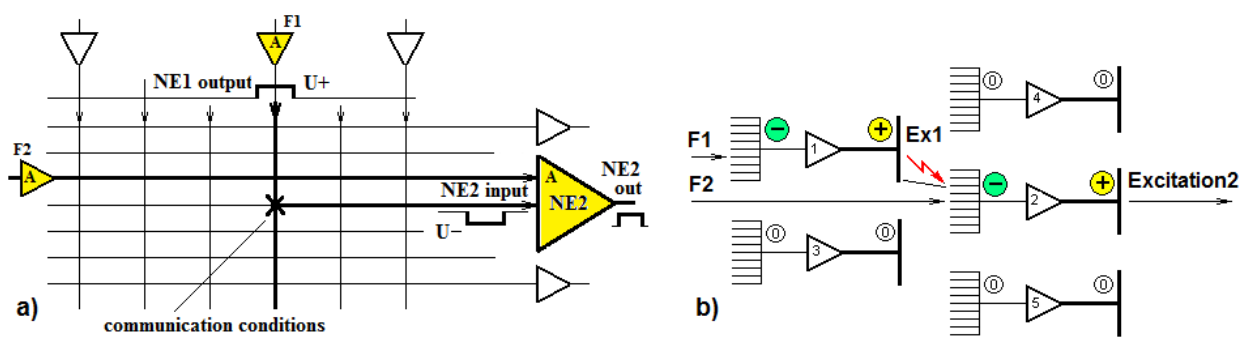

Fig. 4. A scheme for selecting the point of formation of a connection between simultaneously excited neurons.

We assume that the connections between neurons are formed because of the electrical breakdown of the isolation separating the excited neurons. Instead of a breakdown, any other process irreversibly forming a link between excited neurons can act. Moreover, the connections formed must first have the greatest logical weight (the conductivity of the channel arising as a result of electric breakdown). Over time, the conductivity of the channel (connection) naturally decreases because of diffusion-type processes and the influence of the connection decreases. Fading of memory (forgetting) occurs.

The neuron model proposed provides not only propagation of signals and formation of new connections, but also selection of the best ways of the signal propagation by comparing the signal configurations and the weights of the connections of different neurons. It happens as follows. The first to be excited is the neuron, in which the configuration of the coupling weights corresponds best to the configuration of the input signals. By compensating its input and output signals, neuron obstructs excitation of other neurons (which have approximately the same input signal configuration as the newly excited neuron). Therefore, the signal will propagate only along the optimal path, carrying out the optimal reaction corresponding to the configuration of stimuli and to the organism experience, that is, to the weights (forces) of connections between the neurons.

Let us consider what will happen when the situation changes, when the old reaction ceases to be effective. It means that for a long time an irritating signal arrives and continuously excites the neurons of the chain generating an old and ineffective response. As a result, neurons do not have time to restore their working capacity in full. Neurons "get tired". The threshold of their excitation increases. A similar picture of "getting fatigue" is observed with the continuous stimulation of "electric" fish. The potential of the discharges and the magnitude of the amperage decreases each time. As a result of the increase in the threshold of neurons excitation in the chain of the old reaction, it becomes possible to excite neurons adjacent to the old chain. When they are excited, new connections are formed. So the signals move along new paths and a new reaction is formed. It means that because of "getting fatigue", a chain of a new reaction can be formed in the brain. However, this new reaction may also turn out to be ineffective. In this case, the procedure for formation of a new reaction will be repeated. The process will continue until a successful reaction will appear. So neurons "fatigue" allows the brain to change reactions and to be retrained.

Subsequently, if a similar configuration of the input signals occurs, first, a new reaction will be activated, not the old one. This will happen for the reason that the inter-neural connections of the new chain are stronger than the connections of the old chain. The neurons of a new chain win a competition for the exciting signal because the weight of connections is continuously decreasing over time. Therefore, the new connections have the maximum weight (conductivity). It means that forgetting is not a purely negative characteristic.

Thus, in a natural way, the problem of suppression of the old reaction with appearance of a new reaction (reflex) is resolved because of the decrease in the weight of all inter-neural 
connections over time. Ivan P. Pavlov, a physiologist, a Nobel Prize winner, called this suppression as "the damned question of physiology" [4]. Our model easily explains the suppression of old reflexes when new ones appear. Moreover, it does not need to introduce into consideration additional entities (inhibitory neurons and connections). Appearance of inhibitory structures would cause the necessity to centralize control of all neurons with a centralized comparison of the results. Our model also provides the same result because of the existence of competition for the signal between the new stronger and old weaker inter-neuronal connections at the local level.

Thus, our local model allows us to explain all the most important features of biological systems of information processing.

The results stated (the possibility of independent training and retraining) are confirmed in practice by the functioning of the created electronic model of the simplest nervous system composed of 3 electronic neural-like elements. The model created has shown the possibility of self-training and retraining [5].

\section{The emergence of thinking}

How does the thinking process happen in the brain? The more stimuli are taken into account by the brain, the higher are the chances of individual organisms to survive. Therefore, from generation to generation, the number of neuron chains reacting to individual stimuli will increase. These chains will form reactions that are even more complex. The number of connections between neurons will grow, the chains will get longer, will intersect and join each other. Predictions for the arrival of stimuli will become increasingly multi-tiered and an increasing number of factors will be taken into account. Because of the increasing length of the chains and the increase in the number of intersections of the chains, the pathways of the signals from the receptors to the effector become more complex. A signal generated by some receptor can move for a long time through the structures of the brain receiving additional stimuli from signals from other stimuli. Such motion of a signal along the brain structures can be called as the flow of thought through the structures of an intelligence carrier. This carrier can be natural (in humans and animals) or a device, which is an artificial intelligence carrier (AIC). The source carrier itself does not have intelligence. It must be trained. Similarly, a computer becomes useful only after installing the software. Both animals and people train and educate their progeny. After passing the course of primary tuition (education), the bearer of the intellect (a young representative of a man or an animal) receives intellect of a certain level. This level depends on the capabilities of an intelligence carrier and on the quality of instruction. It is especially high among people because of the developed system of training and information transfer from primitive drawings on rocks and cuneiform tablets to modern systems of information transmission and visualization. A man gets the mind.

\section{Conclusion}

In the brain, in contrast to the currently used technical information processing systems, information is fixed not by changing the state of existing elements of the information processing system, but by formation of new elements, which are traces of events. This is how natural memory works, where the organism's experience is fixed. The processing of information in such a system consists in signal passing through already existing brain structures (where the organism's experience is) to effectors (manipulators). Signals are generated in receptors under environmental exposure on the body. These signals enter the brain, pass through its structures in accordance with the experience of the organism and come 
to the effector, which provides an effective reaction of the organism controlled by the brain to the effect of the environment. Signals can fade along the path and not reach the effectors.

When passing through the structures of the brain signals make traces, it means that new connections are formed between the neurons. This is the way how the organism's experience is accumulated.

Such a system for processing input information based on the experience of the organism (the previous information fixed in the brain) is so simple that it is self-organized (self-formed) without an external controlling (directive) influence in each organism with an appropriate carrier based on neurons with the structure and functions proposed in this paper.

The proposed model of a neuron, which is the main structural component of the brain, and simple natural requirements allow us to explain practically all the main features of the brain, which is a natural carrier of the intellect.

Because of the bipolarity of signals at the input and output of the neuron, training (formation of new connections) is provided.

Because of the mutual compensation of bipolar signals, selection of optimal path of signals is ensured, that is an optimal response to configuration of stimuli in accordance with the past experience of the organism.

Because of the "fatigue" of neurons, retraining is provided, that is formation of new links under the conditions of change.

Because of the decrease in the weight of connections over time, forgetting and coming of new reactions to the forefront occurs, that is "inhibition" of old reactions.

Consideration and understanding of the features of functioning of the natural intelligence carrier enables to create an artificial intelligence carrier. Our model shows that this is quite possible with the current level of circuit technologies [6, 7]. To do this, it is sufficient to create a device with a sufficient number of microelectronic neural-like elements (NEs) and to conduct initial training of the artificial intelligence carrier created. In microelectronic structures, it is inconvenient to create large electrical capacitances. Therefore, to ensure formation of bipolar potentials, it seems more convenient to use a circuit with two supply voltages $\mathrm{U}+$ and $\mathrm{U}-$. The pulse output time $\mathrm{PD}$ and the restoration time RT are simulated using mono-vibrators.

Training always occurs based on already existing connections between neurons (knowledge). Therefore, when creating AIC, it is necessary to establish initial connections between neurons in it. These connections are equivalent to the unconditioned reflexes of natural organisms formed in the species of organisms over many generations.

\section{Acknowledgements}

The research presented in this paper was supported by the Russian Foundation for Basic Research (grant No 16-29-04388/17). The authors are grateful to Tatiana B. Rumyantseva from Tomsk State University for English language editing.

\section{References}

[1] V.N. Shumilov, Principles of the brain functioning. Engineer's look. (Ekaterinburg, 2008).

[2] G. Yang, F. Pan, W.-B. Gan, Nature (2009)

[3] G. McCulloch, W. Pitts, Automatic, 363 (1956)

[4] I. P. Pavlov. Brochure of the Academy of Science of the USSR XXVIII, 4 (1932) 
[5] V.N. Voronin, V.N. Shumilov, Yu.A. Shumilov, A.R. Tsoy, A.V. Kiselev, S.M. Sharshov. Report at the 11th International Congress "Neuroscience for Medicine and Psychology", Sudak, (2015).

[6] G.M. Mladenov, V.M. Spivak, E.G. Koleva, A.V. Bogdan, Nanoelectronics URL: http://me.kpi.ua/downloads/Nanoelectronics_1.pdf

[7] V.N. Shumilov, V.I. Syryamkin, M. V. Syryamkin, AIP Conference Proceedings, 1688, 040007 (2015); doi: 10.1063/1.4936040 